\title{
Technical Capability Level 2 Unmanned Aircraft System Traffic Management (UTM) Flight Demonstration: Description and Analysis
}

\author{
Jeffrey Homola, Quang Dao, \\ Lynne Martin, and Joey Mercer \\ NASA Ames Research Center \\ Moffett Field, CA., USA \\ $\{$ jeffrey.r.homola, quang.v.dao, \\ lynne.martin, joey.mercer\}@nasa.gov
}

\author{
Christoph Mohlenbrink and Lauren Claudatos \\ San Jose State University/NASA Ames Research Center \\ Moffett Field, CA., USA \\ \{ christoph.p.mohlenbrink, lauren.e.claudatos \}@nasa.gov
}

\begin{abstract}
NASA's UAS Traffic Management (UTM) project concluded its second flight demonstration activity in late October 2016. This activity demonstrated the capabilities and functionality incorporated into its Technical Capability Level 2 (TCL 2) concept, which envisions future operations that are low density, capable of being performed over sparsely populated areas, and allow for a concurrent mix of longer duration, beyond visual-line-of-sight flights and shorter flights within visual-lineof-sight (VLOS). To incorporate these features into a flight demonstration, a scenario-based approach was taken to address different aspects of the TCL 2 environment and to meet defined objectives. This paper will describe elements of how the flight activity was conducted and present analyses regarding UTM operations, system messages, and alerting as they pertained to meeting the demonstration objectives and shedding light on research questions and lessons learned.
\end{abstract}

Keywords-UAS; UTM; TCL 2; flight demonstration

\section{INTRODUCTION}

Unmanned aircraft systems (UAS) provide operators with the ability to control an aircraft from a remote location or provide strategic guidance when the vehicle is operating autonomously. These systems have a wide range of applications, and their affordability make them an attractive alternative to employing manned aircraft for similar missions. With their growing popularity, sales of all sizes and types of UAS in the United States is expected to grow from 2.5 million in 2016 to 7 million units in 2020 [1]. Based on current projections, the potential UAS fleet size will exceed that of manned aircraft by 35 times in 2020 [2]. NASA's efforts in developing the UAS Traffic Management (UTM) concept and ecosystem offers initial guidance for accommodating the projected demand.

The primary purpose behind NASA's UTM is to safely enable the large-scale commercial application of small UAS (sUAS) (less than 55 pounds) in low altitude (below $500 \mathrm{ft}$ ) airspace $[3,4]$. NASA's plan for research and testing of the UTM concept is a phased approach distinguished by four technical capability levels (TCL) outlined in Figure 1. Of the four, NASA has completed flight demonstrations for TCL 1 and, more recently, TCL 2 [5].

\begin{tabular}{|c|c|c|c|}
\hline & 2 & y 3 & 4 \\
\hline $\begin{array}{l}\text { - Airspace volume } \\
\text { use notification } \\
\text { - Over unpopulated } \\
\text { land or water } \\
\text { - Minimal general } \\
\text { aviation traffic in } \\
\text { area } \\
\text { - Contingencies } \\
\text { handled by UAS } \\
\text { pilot } \\
\text { - Enable agriculture, } \\
\text { firefighting, } \\
\text { infrastructure } \\
\text { monitoring }\end{array}$ & $\begin{array}{l}\text { - Beyond visual line- } \\
\text { of-sight } \\
\text { - Tracking and low } \\
\text { density operations } \\
\text { - Sparsely populated } \\
\text { areas } \\
\text { - Procedures and } \\
\text { "rules-of-the-road" } \\
\text { - Longer range } \\
\text { applications }\end{array}$ & $\begin{array}{l}\text { - Beyond visual line- } \\
\text { of-sight } \\
\text { - Over moderately } \\
\text { populated land } \\
\text { - Some interaction } \\
\text { with manned } \\
\text { aircraft } \\
\text { - Tracking, vehicle- } \\
\text { to-vehicle, internet } \\
\text { connected } \\
\text { - Public safety, } \\
\text { limited package } \\
\text { delivery }\end{array}$ & $\begin{array}{l}\text { - Beyond visual line- } \\
\text { of-sight } \\
\text { - Urban } \\
\text { environments, } \\
\text { higher density } \\
\text { - Autonomous, } \\
\text { vehicle-to-vehicle, } \\
\text { internet connected } \\
\text { - Large-scale } \\
\text { contingencies } \\
\text { mitigation } \\
\text { - News gathering, } \\
\text { deliveries, personal } \\
\text { use }\end{array}$ \\
\hline
\end{tabular}

Fig. 1. UTM Research Technical Capability Levels.

The TCL 1 flight demonstration involved targeted testing of the UTM concept and early architecture. Test flights were conducted such that no more than 2 operations were concurrently active. These operations remained within singular 3D volumes intended to encompass the flight path of the vehicle, which also required the aircraft remain within visualline-of-site (VLOS) throughout its flight. The UTM research platform checked for intersections between different volumes. When submissions were clear of conflicts, a notification was sent to the operator indicating that they were clear to proceed with their operation. However, if a conflict existed, a notification was sent that the submission was rejected. This process of de-conflicting airspace volumes served as an initial test of a safety layer intended to provide strategic separation at the planning stage.

For the TCL 2 flight demonstration, the technical capabilities from TCL 1 were carried over and the concept was extended to include a mixture of VLOS and beyond visualline-of-sight (BVLOS) operations. Additional enhancements included alerting for airspace intrusion, alerts to contingency management, and segmented flight planning that allowed stratification of operational volumes and more efficient use of airspace during BVLOS operations. Conformance monitoring of flight positions and alerting was also a key component that addressed a more tactical layer of safety potentially necessary for enabling BVLOS and operations in close proximity. 
A scenario-based approach was taken to integrate the TCL 2 enhancements and to design interactions that tested the concept, procedures, and technologies. An overall description of the TCL 2 demonstration and the results were presented in [5]. This paper will build upon [5] to provide a more detailed description of the demonstration and present results regarding the overall performance as well as an examination of the safety layers that were incorporated as part of the test.

\section{TCL 2 DEMONSTRATION BACKGROUND}

NASA's UTM project has a well-developed simulations infrastructure and capability [6]. The many variations of operations, volumes, and associated messaging and procedures were developed and extensively tested in a laboratory setting through simulation. However, simulation does not adequately reflect the diversity of concerns and uncertainties that are inherent in the airspace and potentially impactful on operations. Such concerns are critical in gaining awareness and understanding of as the UTM research and development effort continues. Therefore, a live flight demonstration with industry partners from a broad segment of the trade space was planned and executed to observe TCL 2 technologies and operations in a live environment.

\section{A. Test setting and participants}

The flight demonstration was conducted at the Reno-Stead Airport UAS test range in Reno, Nevada. A variety of technologies from industry were deployed at the test site to provide data and services in support of focus areas such as weather and surveillance. The UTM environment at the test range was internet-based and all of the clients developed by the test partners and NASA to perform and support UTM operations connected via wifi or cellular connections. The initial architecture for the TCL 2 research platform was centralized such that all of the UTM clients connected remotely to the core system that was housed at NASA Ames Research Center. Data was collected via the research platform, and interfaces developed for visualizations pulled from the database in real time in order to provide researchers and participants with the opportunity to monitor operations as they unfolded.

Participants came primarily from industry, with a small complement of NASA flight crews. The participants were divided into three separate groups, each with three days dedicated to flight testing, which included setup and proficiency flights as well as the actual data collection runs. The flying partners brought a variety of vehicles to the test. Eleven unique vehicles were flown, each with different performance characteristics that needed to be accounted for in their planned flight profiles. Of those 11 vehicles, six were multi-rotors and five were fixed wing. For a more detailed presentation of the test range, vehicles, and partner technologies, refer to [5].

\section{OBJECTIVES AND SCENARIO-BASED APPROACH}

\section{A. Objectives}

In preparation for the flight test, a number of test objectives were formulated in accordance with the TCL 2 construct. Each of the objectives was designed to address an important feature of the environment envisioned to encompass TCL 2 types of operations. The test objectives developed were as follows:

- BVLOS operations (solo and simultaneous)

- $\quad$ Altitude Stratified operations (VLOS and BVLOS)

- Intruder aircraft tracking and alerting

- $\quad$ Rogue aircraft alerting

- Dynamic re-routing

- $\quad$ Contingency management alerting

- $\quad$ Public Safety operations

- $\quad$ Simulated aircraft

\section{B. Scenarios}

To address the test objectives, four scenarios were developed that consisted of a backstory and associated flights that would achieve the objectives if conducted properly. These scenarios were the drivers for the overall test points conducted during the demonstration. The first of these scenarios was referred to as the "Agriculture" scenario. This was considered to be a warm up scenario as the interactions and missions for each flight were somewhat simpler, yet fully tested a number of functions and met certain objectives. The idea behind this scenario leveraged the use case of small UAS being used in an agricultural and inspection setting in that some of the vehicles were performing flights in line with those that would be performed for crop inspections, railroad inspections, as well as inspections for other key infrastructure components in an agricultural setting.

The second scenario was referred to as the "Lost Hiker" and involved a number of interactions. In this scenario, a number of different types of flights were conducted: cellphone tower inspection, sporting event and news coverage, traffic monitoring, and forest surveying. A simulated lost hiker was reported and a response ensued in which a nearby first responder reserved the airspace surrounding the location of the report through an in-flight plan modification and conducted a search operation. The airspace reservation resulted in notifications to nearby users to remain clear of the area and prevented others from accessing the airspace until the operation was complete.

The third scenario, referred to as the "Ocean" scenario, involved maritime operations. These included oil rig inspections, marine wildlife monitoring, harbor surveillance, and fish and game enforcement operations. Similar to the "Lost Hiker," the need for a water rescue was reported with resulting response. 
The final scenario that was developed was referred to as the "Earthquake" scenario. This scenario involved all flights initially performing various operations (e.g., terrain mapping, supply delivery, inspections) until a simulated earthquake occurred. Following the report of the earthquake, all flights were re-purposed to provide disaster response. This required each flight to submit in-flight plan modifications and begin new operations accordingly as part of the response effort.

Incorporated into each of these scenarios were additional features designed to more fully address the test objectives and exercise UTM functionalities. Figure 2 presents visuals for each of the scenarios where it can be seen that the profiles that were developed included a mix of LOS and BVLOS operations, altitude stratified operations, as well as scripted situations that required in-flight modification. Priority operations were also incorporated into the scenarios as well as intruder aircraft for surveillance tracking. The flight profiles that were developed for each of the operations were designed to prompt interactions between operations, which would also trigger automated messaging and other data exchanges.
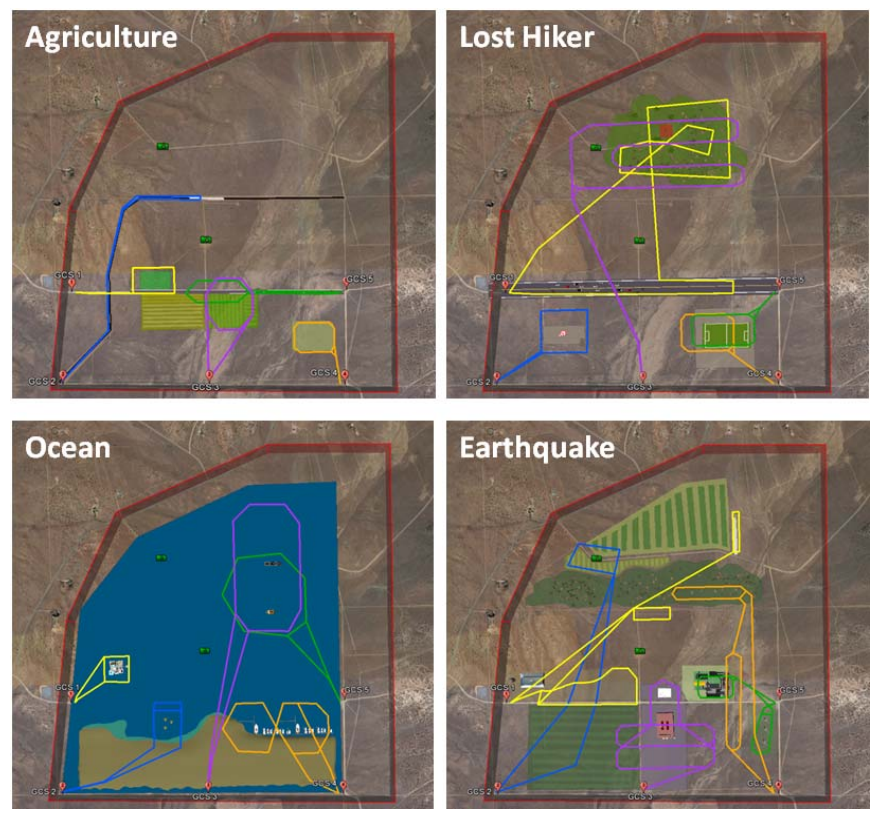

Fig. 2. Four scenarios developed for UTM interactions in a TCL 2 environment

\section{PROCEDURE}

The TCL 2 flight demonstration was divided into three, three-day sessions with different groups of flying partners participating in each session. The format for each session was designed such that the first day was setup, equipment checks, and ground testing at the range. The second day involved safety briefings and proficiency flights early in the flight window. Test card data collection flights were commenced if the proficiency flights were adequately completed and any issues were corrected if necessary. The proficiency flights included a mix of simple VLOS and BVLOS operations that were designed to provide flight crews with familiarization in submitting flight volumes and flying their vehicles within its bounds. Proficiency flight volumes were simple and provided operators with room in which to put their vehicles through a number of checks and to ensure the data exchanges with the UTM research platform were correct and properly handled. VLOS proficiency flights were conducted with multiple simultaneous flights and BVLOS proficiency flights were performed one operation at a time for range safety. The third and final day was typically devoted entirely to data collection runs where different scenarios were performed in succession. The available flight windows each day influenced how many test cards were able to be completed.

At the conclusion of each flight day, all of the individuals involved in the day's events gathered for a debrief and discussion. Participants were provided an opportunity to discuss issues that were of relevance to the test and the other partners and researchers as well. At the conclusion of the debrief, human factors researchers that served as observers (HF-O) were given time to hold separate debrief discussions that were focused on the flight test with respect to the UTM concept and the partners' experience in that context.

\section{A. Roles and Architecture}

Flight crews differed in their size, having between two and five. In five-person teams, each person had a distinct role, while in two-person teams the members worked combined roles. The most common crew size was four people. In addition to the flight crew, there was an Observer Controller (OC) at each ground control station (GCS) that was always in contact with Visual Observers (VO) and the Mission Manager $(\mathrm{MM})$ via radio. The $\mathrm{OC}$ relayed critical information from the MM to the flight crew and VOs to ensure operations were coordinated on the range. During prior testing it was found that the flight crews needed better awareness of their role in a UTM environment. As a result, a UTM representative (UTMrep) was embedded with each flight crew to provide that awareness through visualizations and verbal feedback on an individual flight's performance as a UTM operation. Figure 3 presents a layout of each team's structure as well as the communication pathways.

In the same way that there were a mix of team members and vehicle types, UTM clients that interfaced with the research platform also differed. Users brought eight different clients to the flight test. All of the clients were in various stages of development, so the numbers and types of functions and features varied accordingly. To participate in the demonstration, all clients had to have certain capabilities, including the ability to submit a flight volume request to the UTM system and to receive messages back from the UTM system. Beyond this, partner interaction and client interfaces to meet these functions were very different. During operations, the flight crew was primarily working with their own UTM client and interfaces. In general, UTM messages were provided to the flight crew by the partners' clients. Certain configurations involved the UTMrep relaying UTM messages to the flight crew verbally. 


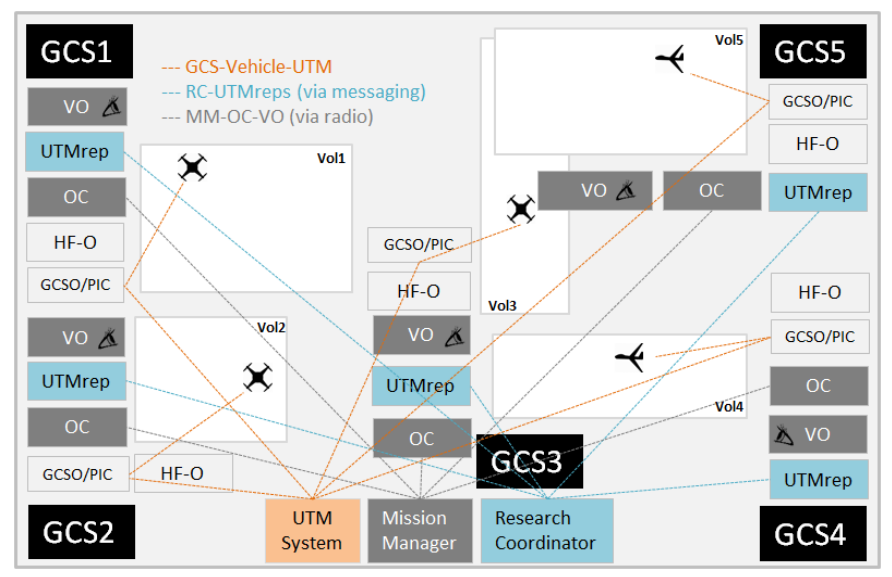

Fig. 3. Architecture and communications flow during the demonstration

\section{OPERATION VOLUMES AND GEOGRAPHIES}

According to projections, the density and complexity of sUAS operations in a future UTM environment will be quite high in certain segments of airspace (e.g., urban centers and surrounding areas). By necessity, therefore, the future system will rely on highly automated functions to monitor and manage operations in a safe, efficient, and effective manner. The traditional approach of air traffic management and control as it applies to manned aviation will not scale appropriately to the operational environments envisioned as part of UTM.

A key operational component that enables airspace management and serves as the reference for the flight and conformance monitoring tested in the demonstration is the Operation Volume. An Operation Volume can be thought of as consisting of multiple Operational Geographies: Flight Geography, Conformance Geography, and Protected Geography (Fig. 4).

The Flight Geography (denoted in green) is what the operator submits that defines the volume of airspace that the operation is intended to remain within, and the times in which the volume will be active. The Conformance Geography (denoted in blue) is a system-provided geography based on the submitted flight. The Conformance Geography is typically greater in size to provide additional buffer and account for environmental or performance uncertainties. The Protected Geography (denoted in red) is an additional system-provided geography that encompasses the Conformance and Flight Geography volumes. The outer bounds of the Protected Geography map to the outer bounds of an Operation Volume. If a vehicle exceeds its Conformance Geography but remains within the area between the Conformance and Protected Geography, the operation will transition from an "Activated" state to "Non-Conforming." The operator can take corrective actions to bring the vehicle back within the bounds of the Conformance Geography. However, if the vehicle remains within the area between the Conformance and Protected Geography for a length of time in excess of a pre-determined threshold, the operation will transition from a "NonConforming" to a "Rogue" state. Additionally, if a vehicle breaches the Protected Geography, the operation will transition to a "Rogue" state and is not expected to be able to regain conformance.

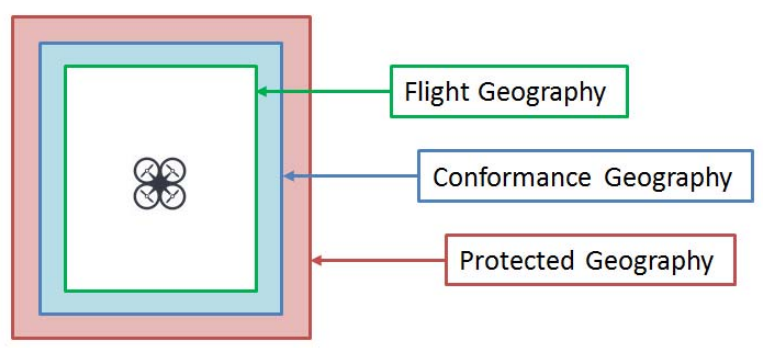

Fig. 4. Operational geographies and conformance monitoring

UTM operations are expected to provide continuous position updates of the vehicle throughout the entirety of the mission. Each update is compared against the different geographies in order to assess the appropriate state. Different messaging responses are triggered according to state transitions. A transition from an 'Activated' to 'NonConforming' state will result in an automated message sent to the operator that the vehicle is no longer in conformance. This provides the operator with time to bring the vehicle back within the bounds of the conformance geography and return to an 'Activated' state. If an operation transitions to a 'Rogue' state, however, not only does the operator receive notification messages, but operators that have vehicles within a certain proximity of the 'Rogue' operation will receive automated messages providing notification and awareness of the situation.

A distinction between what was developed and tested in TCL1 versus TCL 2 with regard to operation volumes is that in TCL 2, a single operation could have multi-segmented volumes, each with different geometric and temporal characteristics. The ability to segment the volumes of an individual operation, particularly for BVLOS operations, provided a means to explore the ways in which airspace can be managed efficiently-an enabler for higher densities of operations envisioned for future TCL environments. The ability to segment volumes also enabled altitude stratified operations where multiple flights could occupy the same lateral volumes of airspace but have safe vertical separation provided by stacked volumes.

\section{RESULTS}

Upon completion of the overall TCL 2 Flight demonstration, there were a total of five data collection days of flight. During that time, all of the data exchanges between partners and the UTM research platform were recorded and stored in a database for post-test analyses. This section will present results of analyses that were performed at two levels of detail. The first presentation will focus on the high level descriptive results that relate to the overall performance of the flight demonstration. The second presentation will focus on more specific results as they relate to flight and operational 
performance in the context of operations in close proximity and the safety layers that enable them.

\section{A. Demonstration Results}

Across the nine days of the flight demonstration, there were a total of five days devoted specifically to flight data collection. Six days of data collection were originally planned (two days per each of the three participant groups), but one day was impacted by high winds that prevented any flights. The available window of flight time varied each day depending primarily on environmental conditions. Winds were constantly monitored and a determination was made as to when the wind speeds exceeded the safe thresholds for the vehicles to operate. The winds were typically too excessive by early afternoon.

Given the available flight windows, a total of 10 full scenarios were completed. The Mission Manager, in coordination with the research team, decided which of the four available scenarios to perform on each of the flight days. Of the 10 data collection runs, the Ocean scenario was performed the most at five times followed by the Lost Hiker and Earthquake scenarios, which were completed twice each. The Agriculture scenario was conducted the least with one completion, which was expected because it was designed more to test proficiency than for full objective testing.

Across the 10 completed scenarios, a preliminary analysis of the data provided the determination that there were 35 live test card flights performed as part of UTM across the three participant groups. Additional simulated flights were conducted as part of the test scenarios while interacting with the UTM research platform, but the analyses presented here were focused on the live flights only.

In relation to the 35 test card flights, each flew for different durations depending on their mission and performance capabilities. When aggregated, these flights operated for a combined duration of 7.66 hours and accumulated 142.61 nautical miles (nm) of along-track distance flown within the UTM environment. The flights were staggered according to their mission/role within the scenarios, which resulted in varying numbers of concurrent operations. Figure 5 presents a visualization for each of the flight days, comprised of the 35 live test flights, in which active flight times were compressed into a continuous duration for a given day. The $\mathrm{x}$-axis represents the time duration of the flights and the $y$-axis represents the number of concurrent operations that were observed during the times of flight. From this perspective, Figure 5 shows that the maximum number of live, concurrent operations during the test scenarios was four. It should be noted, however, that additional simulated flights operated within the UTM environment at the time to raise the number of concurrent operations to five during certain peak periods.

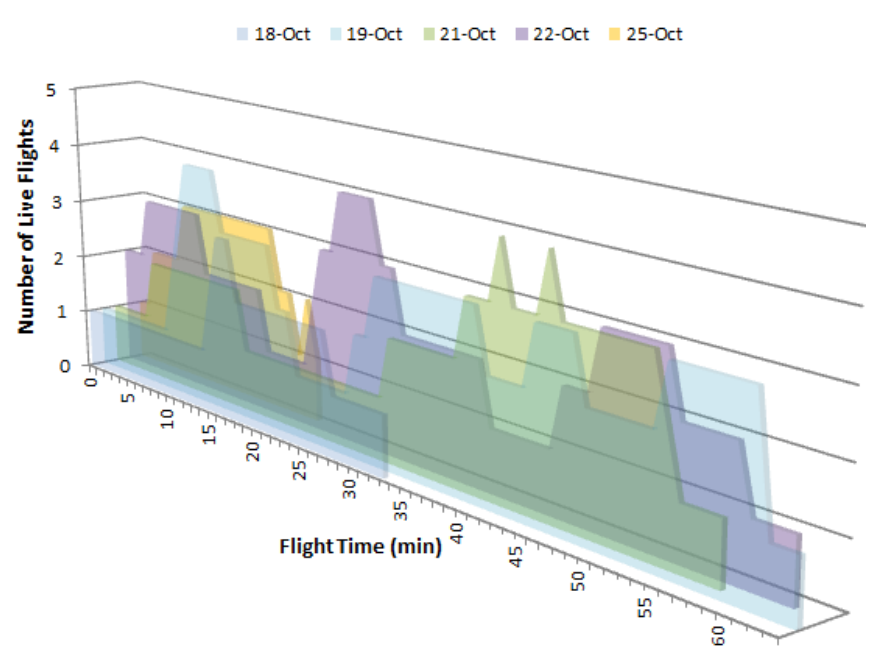

Fig. 5. Aggregate time of flight across all days of data collection

According to the flight profiles designed as part of each test scenario, a mix of distances and altitudes were covered in the course of performing the test missions. Figure 6 presents a scatter plot of the maximum lateral distance flown from launch (x-axis) and the maximum altitude flown (y-axis) for each of the test flights. From the plotted distances, it is evident that there was a mix of VLOS and BVLOS flights, with the furthest lateral distance reached relative to launch location during a BVLOS flight at $8350 \mathrm{ft}(1.37 \mathrm{~nm})$. The altitudes flown were typically between 200 and $500 \mathrm{ft}$ above launch locations, and the maximum altitude reached during data collection was $5619 \mathrm{ft}$ MSL.

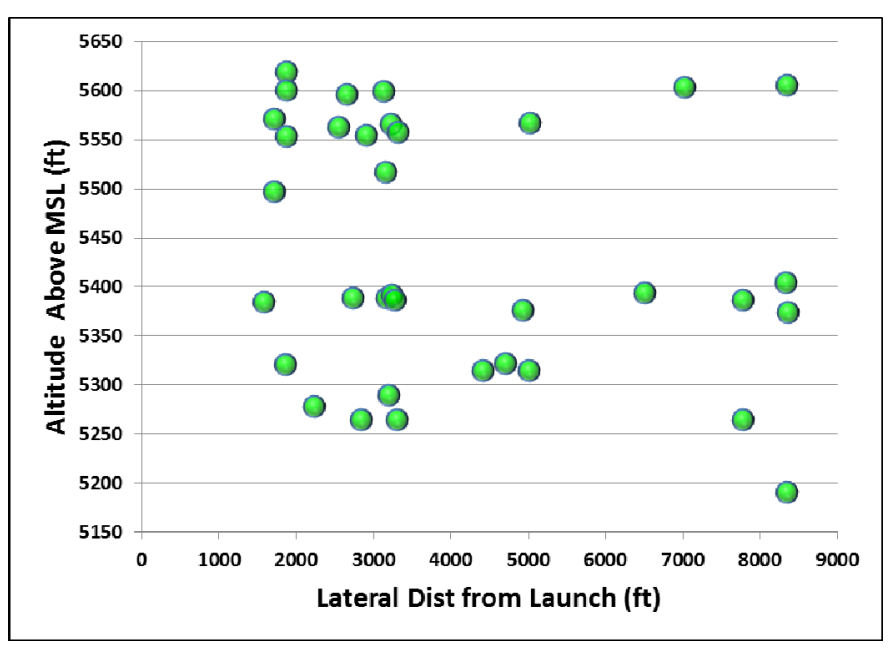

Fig. 6. Diversity of distances and altitudes in which test vehicles operated during the demonstration

The test card scenarios were designed to meet a number of test objectives during the course of their completion. Each of the scenarios addressed a partially different, but not mutually exclusive, set of objectives. To illustrate an example of multiple objectives achieved during a single test scenario, Figure 7 presents a plot in Google Earth of the flight paths as reported into the UTM research platform for each test flight 
along with the associated protected geographies for reference with respect to conformance as observed during one of the Ocean scenario runs.

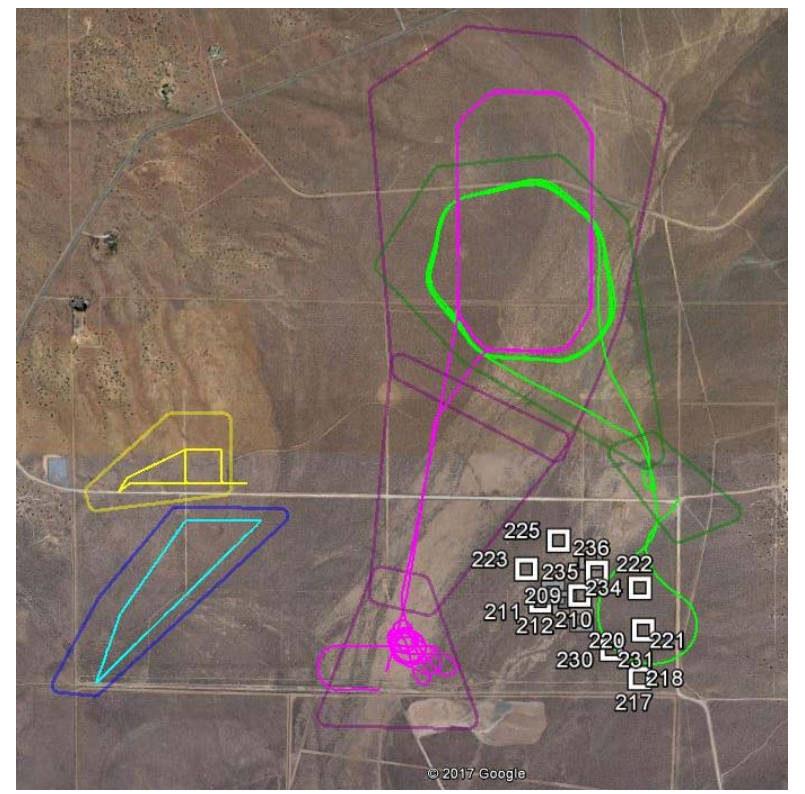

Fig. 7. Plot of flight paths and volumes along with surveillance, illustrating how multiple objectives were achieved during a single Ocean scenario

To deconstruct the operations shown in Figure 7, each of the flights from the different GCS launch locations were plotted in a different color. Beginning with GCS1 in yellow, this particular flight represents a case in which a simulated operation was performed alongside live flights all within the UTM test environment. In this case, the simulated flight breached its protected geography, which transitioned the operation to a 'Rogue' state. Upon this transition, automated messaging was triggered and sent to the GCS1 operator alerting to the 'Rogue' status. Notifications were also sent to the GCS2 operation (in blue) due to its proximity to the 'Rogue' vehicle. The flight from GCS3 (in magenta) represents a multi-segmented BVLOS flight that is also altitude stratified with the flight from GCS5 (in green), which is also multi-segmented and BVLOS. Of note in this example is the fact that both flights experienced unscripted 'Rogue' statuses upon takeoff and landing at GCS5 and GCS3 respectively. This scenario also involved an intruder flight operated from the GCS4 location that tested the surveillance interface between a fielded ground-based radar and the UTM research platform. The vehicle operated from GCS4 intentionally did not submit any operational UTM information to the system, instead relying on the radar to detect and submit return signatures in the form of warning regions within UTM. The white boxes in Figure 7 represent a subset of the warning region data that was exchanged between the radar and the UTM research platform. These regions were visible on the available interface displays and triggered automated notification messages for operations within a prescribed distance such that the GCS3 and GCS5 operators were made aware of a nearby "intruder."

Throughout the course of data collection during the flight demonstration, all objectives were eventually met successfully.

\section{B. System Level Results}

The preceding results have focused on high level, scenario-based events in order to provide an overview of the demonstration and highlight the successful achievement of test objectives. The following results will narrow the focus to more specifically examine system level interactions of flights that were operating in close proximity. As the research progresses from TCL 2 to TCL 3 and beyond, the densities of operations are expected to increase. An understanding of the value that UTM provides in enabling the higher densities and more proximal interactions is important to gain if such applications are to be realized in the future.

\section{1) Closest Point of Approach}

As a means of working toward gaining that understanding, the closest point of approach (CPA) was computed for each pair of flights that operated simultaneously during a given scenario. The analysis was again performed in accordance with the 35 test card flights as the scope of data for comparison. For the calculation of CPAs, the reported position data -latitude, longitude, altitude- for each flight was first organized and sorted by timestamp. For each timestamp in each scenario in which there were multiple flights' position data, the distance between each flight was calculated in turn. The minimum distance was updated for each pair of flights as all timestamps were processed such that the CPA between each flight was known by the end of the run's processing.

Figure 8 presents the results of the CPA analysis as a histogram of distances grouped in $200 \mathrm{ft}$ increments. It should be noted that these results only include the unique distances associated with flight pairs and duplicate distances removed.

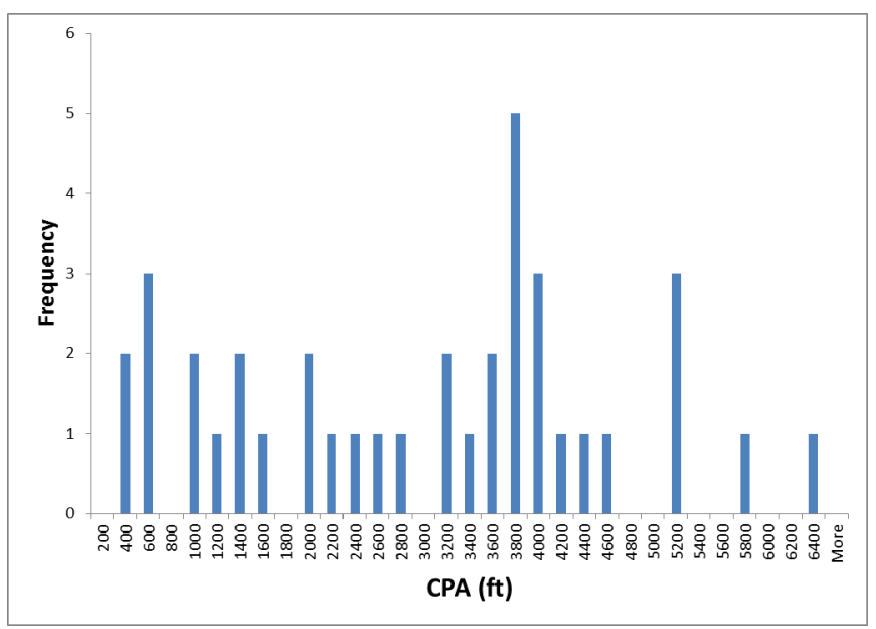

Fig. 8. Histogram of measured closest point of approach between each flight pair per test scenario 
The data presented in Figure 8 shows that there was a wide range of CPA distances observed between flights during the demonstration. The largest distance between flights during a test scenario was $6,287 \mathrm{ft}$, which was between two multirotor vehicles flown from the GCS2 and GCS4 locations in the Agriculture scenario. Conversely, the closest distance between flights was measured at $208 \mathrm{ft}$ between two fixed wing aircraft performing altitude stratified operations as part of the Ocean scenario.

Of the total number of vehicle pairings in which a CPA was computed, seven resulted in distances that were less than $1,000 \mathrm{ft}$. Table 1 presents a description of those instances where it is shown that of the seven cases, five were completed entirely nominally without breaching any of the protected geographies. The concern, however, is if vehicles are brought into close proximity as a result of one or both exiting their protected geography and being in a 'Rogue' state at the time of encounter.

TABLE I. CASES OF VEHICLES APPROACHING WITHIN 1000 FT

\begin{tabular}{|l|l|l|l|l|l|l|}
\hline Scenario & Interaction & CPA (ft) & State-1 & State-2 & Alerts & Operation Type \\
\hline Ocean & GCS3-GCS5 & 208.96 & Active & Active & & Altitude Stratified \\
\hline Ocean & GCS3-GCS5 & 342.33 & Active & Active & & Altitude Stratified \\
\hline Lost Hiker & GCS2-GCS1 & 505.39 & Active & Active & & Lateral Separation \\
\hline Earthquake & GCS4-GCS5 & 533.49 & Active & Rogue & 'RogueNearby' & Lateral Separation \\
\hline Ocean & GCS1-GCS2 & 539.04 & Active & Active & & Lateral Separation \\
\hline Ocean & GCS1-GCS2 & 972.68 & Active & Active & & Lateral Separation \\
\hline Ocean & GCS1-GCS2 & 988.46 & Rogue & Active & 'RogueNearby' & Lateral Separation \\
\hline
\end{tabular}

Two cases out of the seven that came within $1000 \mathrm{ft}$ of each other involved one vehicle that had transitioned to a 'Rogue' state at some point during its mission. The concern with these cases was whether the CPA between the aircraft pair occurred while the vehicle was outside of its volumes. To address this concern, the flight path, volume, and CPA data for each of the involved aircraft were plotted to determine the location of the CPA encounter relative to the reported 'Rogue' positions. Figure 9 presents the results of the plotting and analysis effort where it is shown that, in each case, the vehicles were in lateral conformance at the time of their CPA. The vehicles were in vertical conformance as well, but because the operations were separated laterally, the vertical separation was of a lesser concern. The first case in the left panel of Figure 9 shows that the 'Rogue' state occurred during the landing phase of the GCS5 aircraft (in green) as the vehicle exited the protected geography to the north (shown in red); the CPA location between the two vehicles was well clear of the area in which the GCS5 vehicle became 'Rogue.' The second case shown on the right panel of Figure 9 involved a scripted 'Rogue' encounter in which the GCS1 vehicle intentionally breached its protected geography and hovered for a prescribed time. Even though this was a scripted event, it was still of interest to determine the proximity of the aircraft while one was in a 'Rogue' state. As presented through the plot of data, the CPA encounter occurred well after the GCS1 aircraft was in its 'Rogue' state. In fact, the CPA occurred as the GCS2 aircraft was passing the GCS1 aircraft while in its landing phase.
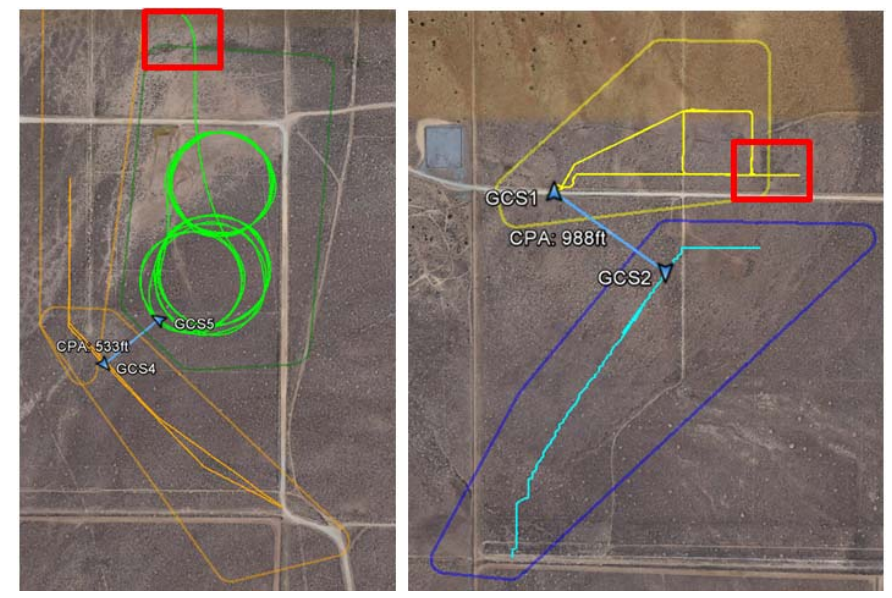

Fig. 9. CPA assessments of aircraft pairs that involved 'Rogue' events

\section{2) Altitude Stratified Operations}

Through the checks of flight geography submissions and conformance monitoring while vehicles are in-flight, operations can be performed in close proximity. As shown in Table 1, the situations in which vehicles were closest were when they were altitude stratified. In a UTM environment, altitude stratified operations refer to operations in which the volumes of two or more aircraft overlap, and the flight paths of the aircraft provide the possibility of overlapping trajectories separated vertically. The performance of altitude stratified operations begins first with the submission of adequately separated flight volumes that account for added buffers. Figure 10 presents the stages in which such operations were conducted during the flight demonstration.
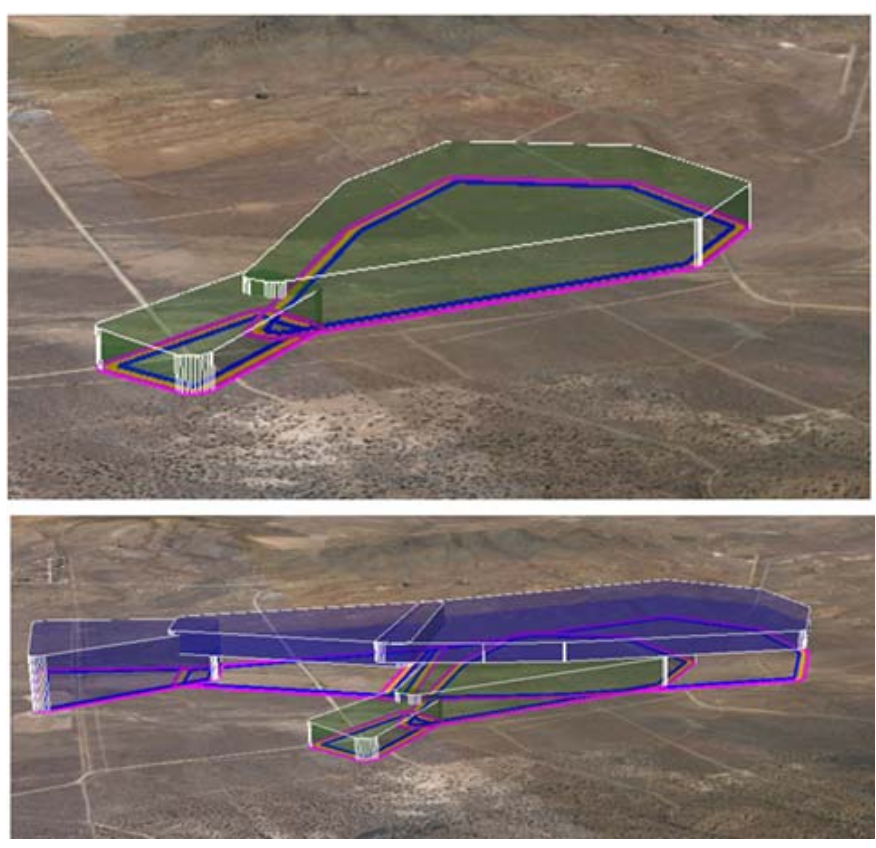

Fig. 10. Example of flight volumes used in altitude stratified operations 
In the top panel of Figure 10, the actual volumes used in an iteration of the Ocean scenario are presented for the lower aircraft of an altitude stratified pair. The segmented volumes used are all clamped to the ground and capped at an altitude that will be clear of the upper aircraft's volume. The bottom panel of Figure 10 shows the volumes (in blue) of the upper aircraft as positioned relative to the lower aircraft's volumes. In this example, the initial volume of the upper aircraft allows for its takeoff and for it to reach an altitude that will clear the lower vehicle's volumes. The remaining volume segments are overlaid above the lower aircraft's acting as a shelf, which provides separation and freedom of movement within the airspace.

\section{3) Safety Layers}

In the TCL 2 environment as constructed in the flight demonstration, there were two primary layers of safety: segregation of operations and conformance monitoring. Segregation of operations was the first layer of safety where flight volumes submitted by operators were supplemented with additional conformance and protected geography buffers and subsequently checked against other operations in the system to ensure that there was no overlap. If an overlap was detected, the submitted operation was rejected. This sequence provided an initial measure of assurance that operations conducted within accepted volumes will be clear of other cooperative operations in the UTM environment.

The second layer of safety that was in place was conformance monitoring. As a vehicle performs its mission as part of UTM, its position updates are continuously checked against the conformance and protected geographies of the operation. If positions are detected between the conformance and protected geographies, the state of the operation transitions to a 'Non-conforming' state and automated messages are sent to the operator's client providing notification of the state change and an opportunity to take corrective measure to resume normal operations. Remaining in a 'Non-conforming' state for an excessive duration or continuing outside of the protected geography results in the operation transitioning to a 'Rogue' state. This state is treated with greater significance because it represents a loss of control or awareness that compromises the safety of operations. Automated messages are again sent to the operator prompting them to close operations, and nearby operators are also sent messages notifying them of a 'Rogue' operation in the area.

An examination of the operations conducted during the flight demonstration that resulted in a reported 'Rogue' status yielded the results presented in Table 2. A total of 12 cases were recorded that were not scripted and did not involve simulated aircraft or 'Rogue' due to delayed position reporting. The cases presented can be categorized according to the way in which the operation became 'Rogue,' which maps to whether the vehicle breached its protected geography vertically or laterally.
TABLE II. SUMMARY OF NON-SCRIPTED 'ROGUE’ EVENTS

\begin{tabular}{|c|c|c|}
\hline Frequency & $\begin{array}{c}\text { Non-scripted Rogue State } \\
\text { Reasons }\end{array}$ & Vehicle Type \\
\hline 4 & Vertical: Too High & $\begin{array}{c}\text { Fixed Wings } \\
\text { Only }\end{array}$ \\
\hline 1 & Vertical: Too Low & $\begin{array}{c}\text { Fixed Wings } \\
\text { Only }\end{array}$ \\
\hline 7 & Lateral & $\begin{array}{c}5 \text { Fixed Wings/ } \\
2 \text { Multi-rotors }\end{array}$ \\
\hline
\end{tabular}

Of the 12 recorded 'Rogue' events, five cases involved a vehicle breaching its protected geography in the vertical dimension. Of that five, four cases involved the vehicle proceeding too high above the geography and a single case in which the vehicle was too low relative to the protected geography. Flight path data was gathered for each of these cases and plotted in profile view as seen in Figure 11. The volumes in which the flight was intended to be within at a given time are represented as blue segments, and the relative position of the vehicle can be compared. For the cases in which the vehicle was high, three of the four appeared to occur shortly after takeoff, suggesting an issue with defining appropriate volumes for fixed wing aircraft to account for that phase of flight. The case in which the vehicle was too low again involved a fixed wing aircraft that breached its protected geographies as it transited from the takeoff volume segment to a segment that had a higher floor.
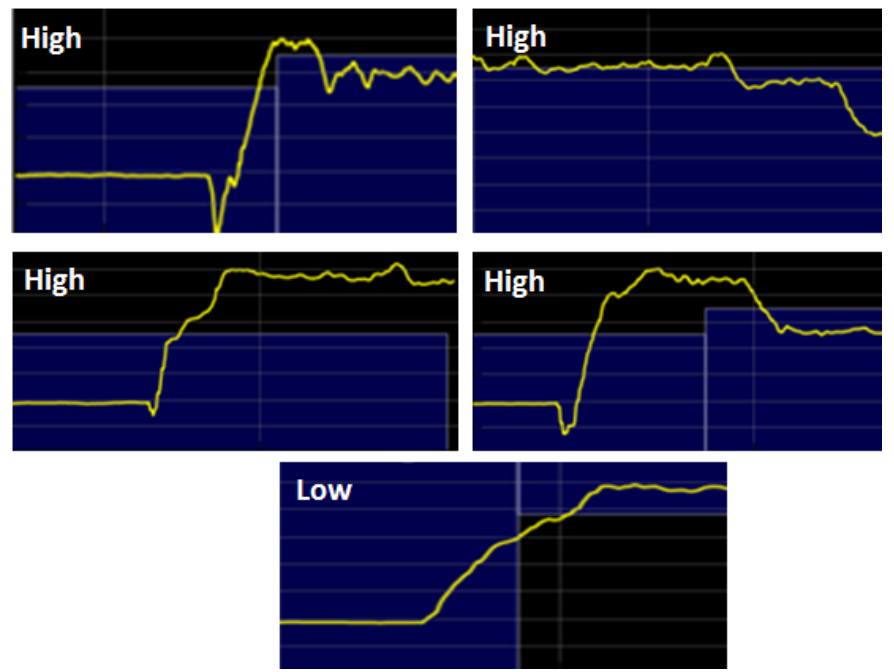

Fig. 11. Profile view of Rogue instances when the vehicle was outside the vertical bounds of its volume (blue segments)

A total of seven 'Rogue' cases were recorded in which a vehicle breached its protected geography laterally. An examination of those cases revealed that the two events involving multi-rotor aircraft appeared to be a result of incorrect flight geographies mistakenly submitted prior to takeoff. The remaining lateral 'Rogue' cases were attributed to fixed wing aircraft. A sample of these events is presented in Figure 12 where the points at which the vehicle breached the protected geography are highlighted with a red box. From this sample, one can see that although there are instances of the vehicle breaching the protected geography during the 
operational phase, most cases involved the fixed wing aircraft exiting the volume either during takeoff or landing. This issue was due to the fact that fixed wing aircraft have wind requirements to adhere to for safe takeoffs and landings, and the volumes that were developed for these flights were not able to account for the dynamic winds a priori.

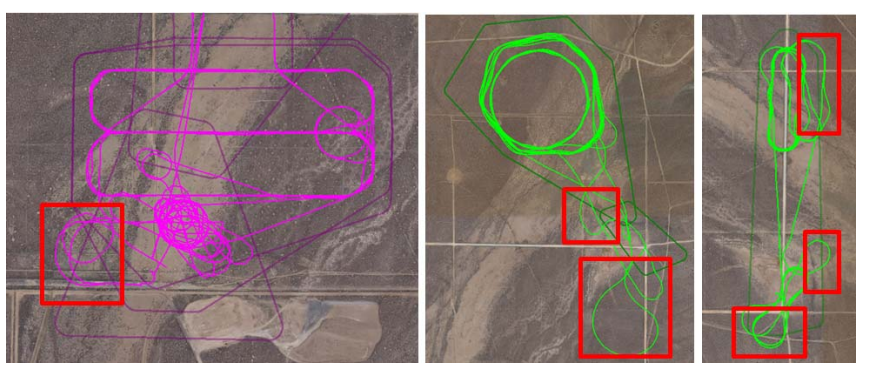

Fig. 12. Rogue instances where the vehicle was outside the lateral bounds of its volume

\section{Operator Feedback}

At the conclusion of each day of flight, the crews and operators that participated gathered for discussion with human factors researchers to review the day's events and to discuss topics relevant to the UTM concept. With respect to the results presented thus far, feedback from these discussions will be presented with regard to experiences with 'Rogue' state and messaging, and situation awareness.

\section{1) 'Rogue' State and Messaging}

For the flight demonstration, the conformance monitoring and state assignments were key components of testing the concept and the research architecture. While it appears as though the detection and notifications of 'Rogue' instances was successful as evidenced from the scripted and nonscripted events, the operators felt as though there would be benefit in providing levels of the 'Rogue' state that were more descriptive and helpful for the operators. The messaging would also help others in close proximity to have a better awareness and understanding of the situation.

Suggestions from crews regarding possible levels of 'Rogue' included:

-Under Control (intentionally outside of its operation volumes and under operator control)

-Under control- lost communications

-Not under control- lost communications

-Rogue due to lost communications with UTM (versus lost communications between GCS and vehicle)

-Rogue due to GCS down

An additional piece of feedback from the debrief discussions was the desire to be able to recover from a 'Rogue' state, similar to how the 'Non-conforming' state is treated if known in advance. However, a valid argument against this is that operators are responsible for being able to account for their vehicle's characteristics and developing operation volumes accordingly.

\section{2) Situation Awareness}

When the flight crew awareness of the operations in the surrounding airspace was low, they spent more time and effort checking their own operations and tried to mitigate their loss of awareness by vigilantly keeping visual contact with the vehicles. Perceived risk as seen in altitude stratified flights and BVLOS flights were also observed to increase vigilance among operators. A NASA-developed mobile application was especially helpful during flight operations in gaining necessary situation awareness particularly in altitude stratified scenarios and in cases where vehicles were operating in close proximity. Reportedly, the most relevant pieces of information for operators were the system state of their own operation and that of that of the altitude stratified operation. The mobile application also helped to build a mental picture of the surrounding traffic, which helped the operators gain an understanding of the surrounding airspace situation

\section{DISCUSSION}

NASA, in close collaboration with industry partners and the Reno-Stead Airport UAS test range, successfully conducted a flight demonstration of the UTM TCL 2 concept. Four scenarios were developed to provide a realistic backdrop to the concept and as a basis for designing interactions between operations and the research platform. These interactions provided a means to address the defined test objectives, which included elements of the TCL 2 environment such as BVLOS flights, altitude stratified operations, Rogue detection and messaging, intruder tracking and alerting, and public safety operations. Over the course of nine days, three separate groups of flying partners and service providers participated. A total of 35 live test card flights were conducted in the process of completing 10 full scenario iterations. The flights included a mix of multi-rotor and fixedwing vehicle types, which were supported by a wide range of GCS and UTM client capabilities and designs.

Through the performance of the scenarios, all of the test objectives were successfully achieved. The test card flights covered a range of distances, altitudes, and durations flown, and were all conducted while connected to the UTM research platform and environment construct. One of the defining features of UTM operations conducted in this environment was the use of multi-segmented operation volumes and their potential application as an initial layer of safety. The multisegment aspect of the operation volumes allowed for testing an approach to more efficient airspace management and flexibility particularly with respect to BVLOS operations. The use of multi-segmented operations also enabled the performance of altitude stratified flights in which vehicles flew within volumes that overlapped laterally but were separated vertically.

With respect to operation volumes being considered a safety layer, the acceptance of volume submissions for operations intended to be conducted in close proximity to one another (e.g., altitude stratified), provided a level of assurance and expectation that the operations are able to be performed 
safely. This assurance applies to the ability to perform BVLOS operations as well where the operation volumes encompass the intended flight path of the vehicle and provides a means to enable awareness of that operation to others in the airspace or to those preparing to enter.

As evidenced by the observation of fixed-wing aircraft unexpectedly climbing in response to thermals reported in [3], and the cases of unscripted 'Rogue' instances reported here, operation volumes are not a guarantee of safety. An additional layer of safety in relation to operation volumes applied in this demonstration, though, was the conformance monitoring of each flight as it submitted position updates to the research platform. In the progression of each of the 'Rogue' cases, as the vehicle first breached its Conformance Geography, an automated notification message was sent to the operator informing them of the breach, with an associated state change to 'Non-conforming.' At this point the operator had a chance to step in and correct the situation, but there was often not enough time or awareness to prevent the breach of the protected geography and resulting 'Rogue' state. Operators received additional messaging upon transitioning to 'Rogue,' although there were suggestions provided through debrief discussions that the messaging would be more valuable if additional detailed information was provided.

An examination of the non-scripted Rogue cases that occurred during the demonstration revealed that, aside from submission errors, the majority of cases occurred during the take-off or landing phase of an operation. It was also notable that these cases all involved a fixed-wing aircraft. From these results, it is clear that more consideration and assistance is needed in terms of designing operation volumes that can account for changing wind directions and vehicle characteristics.

With operation volume de-confliction and conformance monitoring serving as safety layers within the tested UTM environment, there was a high level of confidence in designing interactions that covered a range of operations with varying levels of proximity. The two operations that resulted in the closest encounter -less than $500 \mathrm{ft}$ - were altitude stratified where the vehicles overlapped almost directly as they crossed paths laterally but were separated vertically. Each of those operations was performed nominally, staying within their operation volumes for the entirety of flight. The two cases in which a 'Rogue' state was detected as a result of conformance monitoring and the vehicles had a CPA of less than $1000 \mathrm{ft}$ were shown to trigger the correct messages to the correct operators in close proximity. Situation awareness via client applications was critical to providing operators with the ability to react appropriately with a contingency procedure should the need have arisen.

As the research and environment of focus transitions from a low density TCL 2 environment to one with moderate density near people, additional or different safety layers will be required. Sense and Avoid technologies and the necessary communications mechanisms will likely come to the forefront in enabling the types of operations envisioned for TCL3 and beyond.

\section{CONCLUSION}

NASA was able to successfully demonstrate complex UTM operations in a TCL 2 environment in collaboration with industry partners and the Reno-Stead Airport UAS test range. A scenario-based approach to the demonstration provided a relevant means through which the key elements and objectives of the test could be captured. Although issues were identified through the testing and feedback of test participants, the overall results were in line with the overall UTM objective of safely enabling large-scale UAS operations in low altitude airspace.

\section{ACKNOWLEDGMENT}

The authors would like to thank the many researchers and developers on the UTM team that supported and enabled this work. This research was funded by the Safe Autonomous Systems Operations (SASO) Project of NASA's Airspace Operations and Safety Program (AOSP).

\section{REFERENCES}

[1] FAA. (2016). FAA Aerospace Forecasts. http://doi.org/10.1017/CBO9781107415324.004

[2] Unmanned Aircraft Systems (UAS) Service Demand 2015-2035 Literature Review \& Projections of Future Usage. (2013). Cambridge, MA.

[3] Kopardekar, P., Rios, J., Prevot, T., Johnson, M., Jung, J., \& Robinson, J. E. I. (2016). Unmanned Aircraft System Traffic Management (UTM) Concept of Operations. AIAA Aviation, Technology, Integration, and Operations Conference.

[4] Prevot, T., Homola, J., \& Mercer, J. (2016). From Rural to Urban Environments: Human/Systems Simulation Research for Low Altitude UAS Traffic Management (UTM). 16th AIAA Aviation Technology, Integration, and Operations Conference. Washington D.C. 13-17 June, (June), 1-13. http://doi.org/10.2514/6.2016-3291

[5] Johnson, M. et al. (2017). Flight Test Evaluation of a Traffic Management Concept for Unmanned Aircraft Systems in a Rural Environment. In Twelfth USA/Europe Air Traffic Management Research and Development Seminar (ATM2017).

[6] Homola, J., Prevot, T., Mercer, J., Bienert, N., \& Gabriel, C. (2016). UAS Traffic Management (UTM) Simulation Capabilities and Laboratory Environment, IEEE-DASC-September 26-29, 2016 Sacramento, CA 